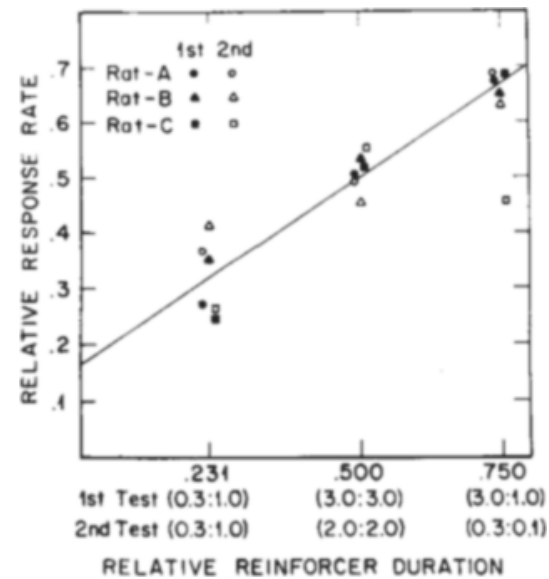

Fig. 2. Relative response rate as a function of relative reinforcer duration for the left bar. The points are means for the last three sessions of the condition indicated. There are separate points for the first and second time a given value of relative reinforcer duration was tested for each rat. The line is a visual fit.

With regard to the effects of changes in reinforcer duration on relative response rate (rate of one response divided by the sum of both response rates), Fig. 2 shows that relative rate of response was a linear function of relative reinforcer duration for the three values tested. By comparing the points on Fig. 2 with the corresponding absolute response rates in Fig. 1, it is obvious that the degree of matching obtained did not come about because of a consistent relation between the absolute rate of a response and the absolute duration of its reinforcer. Rather, as mentioned above, the rate of a response reinforced by a given reinforcer duration varied considerably as a function of the duration of the alternative reinforcer. Whether the orderly relation between relative response rate and relative reinforcer duration depends on these variations, or vice versa, needs further investigation.

\section{REFERENCES}

CATANIA, A. C. Concurrent performances: A baseline for the study of reinforcement magnitude. Journal of the Experimental Analysis of Behavior, 1963,6, 299-300.

RACHLIN, H., \& BAUM, W. M. Response rate as a function of amount of reinforcement for a signaled concurrent response. Journal of Experimental Analysis of Behavior, 1969, 12, 11-16.

WALKER, S. F., HURWTTZ, H. M. B., \& SCHNELLE, J. F. Rates of concurrent responses and reinforcer duration. Psychonomic Science, 1970, 21, 29-31.

\title{
Frustration, competing responses, and error making
}

\author{
JAMES L. BRUNING, Ohio University, Athens, Ohio 45701 \\ RONALD R. SCHMECK, Southern Illinois University, Carbondale, Ill. 62901 \\ and \\ AVRUM I. SILVER, Ohio University, Athens, Ohio 45701
}

The effects of competing responses on performance were examined in a simple T-maze where the number of competing responses inherent to the apparatus and situation were minimal, but where their occurrence could be manipulated. Following reversal training, Ss were frustrated just prior to the choice point of the T-maze. The results supported the prediction that frustrative nonreward would increase the general drive level which would, in turn, lead to greater frequency of choice errors.

While the interaction of task variables and emotionally produced drive has been discussed in relation to anxiety, stress, fear, and other hypothesized internal states, the topic has not been dealt with to any extent in the case of frustration. The general finding for frustration, as has been well documented by the animal research of Amsel (1962) and many others, is that running speed in an alley increases following frustrative nonreward. The same finding has been reported for humans, especially children (Ryan \& Watson, 1968), when lever movement or pressure exerted serves as the dependent variable. As is apparent, however, all of these studies have employed a simple task of running in a straight alley or moving a lever where relatively few competing responses are present.

Studies conducted recently by Schmeck \& Bruning $(1968,1970)$, however, have demonstrated that when the task is complex and there is a relatively large number of competing responses present, the results have not shown the typical enhancement of performance.

In these studies, the basic assumptions and hypotheses were derived from the Hull-Spence model, which assumes that an increase in drive (here assumed to be due to frustration) tends to move the normal probability distribution of momentary $E$ values for all response tendencies further above the $\mathrm{L}$ or threshold. In the instances where there are few, if any, competing responses (usually defined as a simple task), the frustration-produced increment in drive would be expected to raise only the correct tendency, thereby enhancing performance. This would be the case in the running-speed data reported by Amsel where animals perform a simple running response, and, in addition, are usually pretrained before the introduction of the nonreward-frustration trials. However, when competing responses are present in large numbers and are close to the strength of the correct response, the increase in drive would raise both the correct and incorrect tendencies into competitive status, thus increasing the probability that one of the competing responses would occur in place of the correct response.

In the following experiment, the effects of competing responses on performance were examined in a simple T-maze where the number of competing responses inherent to the apparatus and situation were minimal, but where their occurrence could be manipulated. Thus, the basic hypothesis was that frustrative nonreward would increase the general drive level of the $S$, which would, in turn, lead to greater occurrence of a competing response, which was known to be high in the hierarchy.

\section{PROCEDURE}

Thirty-nine hooded rats were maintained on a 23-h deprivation schedule throughout the experiment. The apparatus was a T-maze constructed so that there were three goalboxes. The first goalbox was located in the stem of the maze just prior to the choice point, while the second and third goalboxes were placed at the end of each of the arms in the usual fashion. During Phase 1, all Ss ran to the first goalbox which contained two Noyes pellets. After the S had eaten the pellets, the second start door was opened, and the $S$ was required to make a position choice to receive reward in one of the arms of the T. To control for possible position preference, 19 of the Ss were rewarded in the right arm of the maze and 20 were rewarded in the left. Ss were trained in this fashion until they had learned the correct choice to a criterion of 19 of 20 . Following the learning to criterion, Ss were divided into three groups of 13 each. During Phase 2, Ss continued to receive $100 \%$ reinforcement in the first goalbox, but the arm containing the second reinforcement was reversed. Trials were continued until Ss learned the reversal to a criterion of 5 of 5 . Phase 3 represented the critical trials in which the Ss in Groups 1 and 2 were given 10 additional trials under the following conditions. Ss in Group 1 received 50\% reinforcement in the first goalbox, while Ss 
in Group 2 continued to receive $100 \%$. For Phase 3 , both arms were baited so that regardless of the choice, $S$ received reinforcement in the arm of the maze. The basic prediction was that the introduction of frustration at the choice point would increase the drive level of Group 1 and thus result in the raising of the response originally learned during Phase 1 into competitive status. Consequently, $S$ s in Group 1 were expected to show an increase in choice errors as compared with Group 2, which was not frustrated. In order to determine if the effects of frustration per se might not be somehow related to behavior variability (which in a two-choice situation would be scored as increased error making), an additional control group was included in which original training during Phase 1 was given in the same manner as Groups 1 and 2. Following the learning to the 19 of 20 criterion, however, the reversal training was omitted, and the Ss in Group 3 were immediately subjected to the Phase 3 condition where they received 50\% reinforcement in Goalbox 1 for 10 trials.

\section{RESULTS AND DISCUSSION}

The results were recorded in terms of the number of errors made by each $S$ during Phase 3 . The analysis of variance showed that $S s$ in the reversal-frustrative group (Group 1) made significantly more choice errors (Group 1 mean $=2.8$ ) than the reversal-nonfrustrated group (Group 2 mean $=1.6$ ) and the nonreversal-frustrated control group (Group 3 mean $=.9$ ) $(F=8.43, \mathrm{df}=2,36)$. The result of the Duncan range test comparisons showed that all groups differed significantly from each other, $\mathrm{p}<.001$.

The results of this study demonstrate that the motivational increase resulting from frustration impairs performance when there are competing responses present. In addition, this study suggests the possibility of a more parsimonious interpretation of the concept of clinically defined regression. The implications are that frustration may raise responses leamed at an earlier stage into competitive status and thus result in their occurrence (also, see Ross, 1964).

In addition to generally raising responses above threshold, frustration may also have cue or directional properties. As possible support for this suggestion is a study recently completed by Yaremko, Johnson, Maddox, \& Leckart ${ }^{1}$ where the relationship between choice behavior under noxious conditions induced by shock was compared to later performance when frustrative nonreward was introduced.
Their findings show that inducement of frustration tends to result in behaviors learned previously under another type of stress condition. Consequently, if an experimental analogue of "regression" is to be suggested, it may have to include both the enhancing and cue effects of frustration.

\section{REFERENCES}

AMSEL, A. Frustrative nonreward in partial reinforcement and discrimination learning: Some recent history and a theoretical extension. Psychological Review, 1962, 69, 306-328.

ROSS, R, R. Positive and negative partial-reinforcement extinction effects carried through continuous reinforcement, changed motivation, and changed response. Journal of Experimental Psychology, 1964, 68, 492-502.

RYAN, T. J., \& WATSON, P. Frustrative nonreward theory applied to children's behavior. Psychological Bulletin, 1968, 69, 111-125.

SCHMECK, R. R., \& BRUNING, J. L. Frustration theory and quality of performance: Elicitation and elimination of competing responses. Psychological Reports, $1970,26,987-994$.

SCHMECK, R. R., \& BRUNING, J. L. Task difficulty and the frustration effect. Joumal of Experimental Psychology, 1968, 78, 516-520. NOTE

1. Yaremko, R. M., Johnson, S., Maddox, B. \& Leckart, B. T. Effects of stress during acquisition on T-maze choice behavior during extinction. Unpublished manuscript. 